\title{
Conhecimentos e práticas de enfermagem na prevenção e cuidado às lesões por pressão
}

Knowledge and practices of nursing professionals in pressure injury prevention and care

Conocimientos y prácticas de enfermería en la prevención y atención de las úlceras por presión

\section{Francisca Débora Veras Silva', Priscila Cristina da Silva Thiengo de Andrade ${ }^{\mathrm{II}}$, Eugenio Fuentes Pérez Júnior ${ }^{\mathrm{III}}$, Ariane da Silva Pires ${ }^{\mathrm{IV}}$, Cristiane Helena Gallasch ${ }^{\mathrm{V}}$}

\begin{abstract}
Resumo: Objetivo: identificar o conhecimento da equipe de enfermagem sobre os múltiplos fatores que predispõem ao desenvolvimento e implicam no cuidado de lesões por pressão em pacientes internados em unidades clínicas. Método: estudo descritivo, de abordagem mista, com dados coletados entre abril e julho de 2018 em um hospital universitário do Rio de Janeiro, com 47 profissionais de enfermagem. Após aprovação pelo Comitê de Ética em Pesquisa, aplicou-se o Instrumento para Identificação do Nível de Conhecimento Sobre Úlcera de Pressão e Medidas Preventivas, com resultados analisados por estatística descritiva e inferencial. Posteriormente, realizou-se a entrevista semiestruturada, analisada por meio de análise de conteúdo. Resultados: identificou-se déficit de conhecimento da equipe de enfermagem voltada à prevenção de lesões. Em relação aos cuidados, constatou-se que são prejudicados pela falta de recursos humanos e materiais. Conclusão: faz-se necessária a educação permanente em serviço sobre o tema, além da garantia de recursos humanos e materiais.
\end{abstract}

Descritores: Cuidados de enfermagem; Lesão por pressão; Conhecimento

\begin{abstract}
Objective: to identify the knowledge of the nursing staff about multiple factors that may predispose to development and imply to the care of pressure ulcers in hospitalized patients admitted in clinical units. Method: descriptive study with mixed method, with data collected between 2018 April and July, in a university hospital in Rio
\end{abstract}

IEnfermeira. Especialista em Enfermagem em Clínica Médica pela Faculdade de Enfermagem da Universidade do Estado do Rio de Janeiro UERJ. Rio de Janeiro, RJ, Brasil. E-mail: deby_veras@hotmail.com. ORCID: https://orcid.org/0000-0001-5261-3181

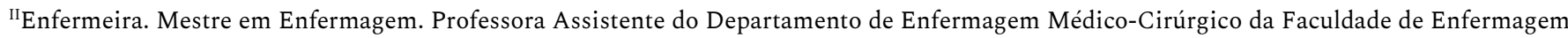
da Universidade do Estado do Rio de Janeiro - UERJ. Rio de Janeiro, RJ, Brasil. E-mail: profprithiengo@gmail.com. ORCID: https://orcid.org/0000-0003-0840-4838

IIIEnfermeiro. Doutor em Enfermagem. Professor Assistente do Departamento de Enfermagem Médico-Cirúrgico da Faculdade de Enfermagem da Universidade do Estado do Rio de Janeiro - UERJ. Rio de Janeiro, RJ, Brasil. E-mail: eugenioperezjunior@gmail.com. ORCID: https://orcid.org/0000-0003-4611-0443

${ }^{\mathrm{IV}}$ Enfermeira. Doutora em Enfermagem. Professora Assistente do Departamento de Enfermagem Médico-Cirúrgico da Faculdade de Enfermagem da Universidade do Estado do Rio de Janeiro - UERJ. Rio de Janeiro, RJ, Brasil. E-mail: arianepires@oi.com.br. ORCID: https://orcid.org/0000-0003-1123-493X

${ }^{\vee}$ Enfermeira. Doutora em Enfermagem. Professora Adjunta do Departamento de Enfermagem Médico-Cirúrgico da Faculdade de Enfermagem da Universidade do Estado do Rio de Janeiro - UERJ. Rio de Janeiro, RJ, Brasil. E-mail: cristiane.gallasch@gmail.com. ORCID: https://orcid.org/0000-0002-0823-0818 
de Janeiro, with 47 nursing professionals. After approval by the Research Ethics Committee, the tool "Instrumento para Identificação do Nível de Conhecimento Sobre Úlcera de Pressão e Medidas Preventivas” was applied, with results analyzed by descriptive and inferential statistics. Latter, participants answered a semi-structured interview, evaluated through content analysis. Results: a knowledge deficit related to injury prevention was identified. Regarding care, there is prejudice related to the lack of human and material resources. Conclusion: permanent inservice education about these issues are necessary, as well as the guarantee of human and material resources.

Descriptors: Nursing care; Pressure ulcer; Knowledge

Resumén: Objetivo: identificar el conocimiento del personal de enfermería sobre los múltiples factores que pueden predisponer al desarrollo e implicar en el cuidado de las úlceras por presión en pacientes hospitalizados en unidades clínicas. Método: estudio descriptivo, método mixto y datos recopilados entre abril y julio de 2018, en un hospital de Río de Janeiro, con 47 profesionales. Después de la aprobación del Comité de Ética en Investigación, se aplicó la herramienta "Instrumento para Identificação do Nível de Conhecimento Sobre Úlcera de Pressão e Medidas Preventivas”. Resultados se analizaron mediante estadística descriptiva e inferencial. Posteriormente, respondieron una entrevista semiestructurada, evaluada a través del análisis de contenido. Resultados: se identificó un déficit de conocimiento relacionado con la prevención de lesiones. Hay prejuicios relacionados con la falta de recursos humanos y materiales. Conclusión: la educación permanente en el servicio sobre estos temas es necesaria, así como la garantía de recursos humanos y materiales.

Descriptores: Atención de enfermería; Úlcera por presión; Conocimiento

\section{Introdução}

O presente estudo tem como objeto o conhecimento da equipe de enfermagem sobre os fatores que predispõem o desenvolvimento de Lesão por Pressão (LP), assim como os cuidados envolvidos em pacientes internados em unidade de internação clínica.

A LP caracteriza-se pelo dano na pele e/ou tecidos moles subjacentes, geralmente sobre proeminência óssea, ou relacionada ao uso de dispositivos. Pode apresentar-se com pele íntegra ou úlcera aberta. ${ }^{1}$ Em 2016, National Pressure Ulcer Advisory Panel (NPUAP), definiu o termo lesão por pressão, que descreve com precisão lesões em peles intactas ou ulceradas, com alterações na sua classificação, substituição do termo categoria, por estágio, e remoção do termo suspeita. ${ }^{1-2}$

Seu desenvolvimento está associado a fatores etiológicos relacionados à intensidade e à duração da pressão no tecido, além de fatores extrínsecos, como fricção, cisalhamento e umidade, e intrínsecos, como idade, condição nutricional e perfusão tecidual. ${ }^{3}$ 
A ocorrência de LP é considerada um problema de saúde pública. Tem despertado preocupação de enfermeiros e da equipe multidisciplinar, visto que sua incidência e especificidade majoram o sofrimento do paciente, além de dificultar a recuperação e prolongar o tempo de internação. ${ }^{4}$ Mediante a presença da lesão, é necessário reavaliar os fatores de risco, com visão ampla dos fenômenos que estão envolvidos e direcionar ações efetivas de acordo com as necessidades identificadas. ${ }^{5}$

Apesar dos estudos sobre as ações voltadas à prevenção e os cuidados realizados após a instalação deste agravo continuarem avançando, na prática assistencial, assim como em estudos científicos, ainda encontra-se baixa adesão às recomendações voltadas para a avaliação, prevenção e tratamento das LP. ${ }^{6}$

O presente estudo refere-se à continuidade de investigação de campo anterior que indicou que, mesmo diante do risco identificado para desenvolvimento de lesões, as mesmas continuavam se desenvolvendo em pacientes internados em uma unidade de internação clínica, sendo necessário identificar os motivos que levavam a tal desfecho para embasar as ações de educação e gerenciamento local. ${ }^{7}$ Além disso, recentemente, o local foi designado para projeto piloto de prevenção de LP, pela Comissão de Segurança da instituição. Assim, questiona-se: quais os conhecimentos que a equipe de enfermagem possui em relação aos fatores que predispõem ao desenvolvimento e aos cuidados com as LP?

É descrito que profissionais de enfermagem, muitas vezes, conhecem as medidas de prevenção de tais lesões, porém há necessidade de reforçar em seu cotidiano as boas práticas baseadas em evidências científicas para garantir a implementação dessas ações. ${ }^{8}$ Ações de educação permanente podem, ainda, promover a adoção de medidas sistematizadas focadas na individualidade de cada paciente. ${ }^{9}$ Destaca-se, ainda, que considerando as metas internacionais de segurança do paciente, as LP estão entre os eventos adversos considerados como prioridade de ação. ${ }^{10}$ 
Conhecimentos e práticas de enfermagem na prevenção e cuidado às lesões por pressão $\mid 4$

Assim, o presente estudo teve como objetivo identificar o conhecimento da equipe de enfermagem sobre os múltiplos fatores que predispõem ao desenvolvimento e implicam no cuidado de lesões por pressão em pacientes internados em unidades clínicas.

\section{Método}

Trata-se de um estudo descritivo, com abordagem mista, integrando dados quantitativos e qualitativos, coletados sequencialmente, ${ }^{11}$ em unidades de internação clínica de um hospital estadual universitário de nível quaternário, no município do Rio de Janeiro. A coleta de dados ocorreu entre abril e julho de 2018.

Foram selecionadas três unidades de internação clínica, que possuíam entre dez e doze leitos, femininos e masculinos, abertos para pacientes no período do estudo. Tais unidades prestam assistência em casos da área de clínica médica, a pacientes maiores de 18 anos, com diagnóstico de doenças cardiovasculares, renais, gastrointestinais, dermatológicas e onco-hematológicas.

Incluindo as três unidades, a equipe de enfermagem era composta por cinco enfermeiros e 51 técnicos de enfermagem, atuantes em plantões diurno e noturno, com média de três técnicos de enfermagem e um enfermeiro no plantão diurno e três técnicos de enfermagem no plantão noturno. Todos trabalham em escalas de 12 horas por 60 horas na referida instituição.

Todos os profissionais de enfermagem foram convidados a participar do estudo, entretanto cinco enfermeiros e 42 técnicos de enfermagem, dos plantões diurno e noturno, aceitaram participar voluntariamente. Foram excluídos seis técnicos de enfermagem que se encontravam em algum tipo de licença ou afastamento. Houve três casos de recusa.

A coleta dos dados ocorreu em duas etapas: a primeira realizada com 47 profissionais da equipe de enfermagem, quando foi aplicado o Instrumento para Identificação do Nível de Conhecimento Sobre Úlcera de Pressão e Medidas Preventivas, composto por 41 questões 
5 | Silva FDV; Andrade PCST, EFP Pérez Júnior, Pires AS, Gallasch CH

objetivas com objetivo de avaliar o conhecimento dos profissionais de enfermagem sobre a classificação, a avaliação dos riscos e as medidas preventivas ao desenvolvimento de LP. ${ }^{12}$

O referido instrumento foi adaptado para o contexto brasileiro e avaliado quanto ao seu conteúdo como parte das atividades da Tese de Doutorado "Efeitos de intervenções educativas no conhecimento e práticas de profissionais de enfermagem e na incidência de úlcera de pressão em centro de terapia intensiva”, em 2006. Destaca-se que a versão original foi desenvolvida em 1995, com o objetivo de investigar o índice de acertos/erros relacionados ao conhecimento sobre prevenção e estadiamento de LP. ${ }^{13}$

Em um segundo momento, foi realizada uma entrevista semiestruturada, composta por três perguntas abertas: “O que você sabe sobre medidas preventivas à lesão por pressão?”, “Como sua rotina de trabalho de enfermagem influencia a prevenção de lesões por pressão?” e "Como a atuação dos demais membros da equipe multiprofissional (ex.: médicos, nutricionistas ou fisioterapeutas) pode contribuir na prevenção de lesões por pressão?”

Participaram desta fase, cinco enfermeiros e seis técnicos de enfermagem atuantes nas três unidades de internação clínica, nos períodos de plantão diurno e noturno definidos por conveniência, a partir da amostra composta na primeira fase. As entrevistas foram realizadas dentro da instituição, em local reservado escolhido pelo participante, durante seu turno de trabalho e duraram em torno de 30 minutos sendo gravadas em mídia digital.

Destaca-se que foi atribuído o mesmo peso aos dados provenientes das duas abordagens, optando pela apresentação separada dos mesmos. Porém, houve um direcionamento da análise qualitativa, a partir das lacunas de conhecimento observadas na aplicação do Instrumento para Identificação do Nível de Conhecimento Sobre Úlcera de Pressão e Medidas Preventiva, na fase quantitativa.

Os dados quantitativos coletados a partir do Instrumento para Identificação do Nível de Conhecimento Sobre Úlcera de Pressão e Medidas Preventivas, foram tabulados no software 


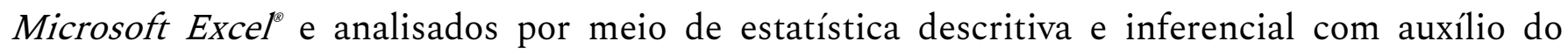
software Statistical Package for Social Sciences ${ }^{\oplus}$ (SPSS), utilizando-se o teste de Qui-quadrado para investigar possíveis associações entre as categorias profissionais e as respostas encontradas, assim como entre as unidades de trabalho $(1,2$ ou 3$)$ e as respostas encontradas $(\mathrm{p}<0,05)$.

Os dados provenientes das entrevistas foram transcritos e submetidos à dupla checagem. Após, foi realizada análise de conteúdo, obedecendo à metodologia composta por: (1) préanálise, com organização do material e composição do corpus do estudo; (2) exploração do material processo pelo qual os dados são transformados sistematicamente e agregados em unidades; e (3) tratamento dos resultados, com inferência e interpretação, buscando embasar as análises e dar sentido à interpretação, ${ }^{14}$ emergindo 166 unidades de registro (UR), 17 unidades de significação (US) e duas categorias: Medidas preventivas de lesões por pressão e Fatores que influenciam no aparecimento de lesões por pressão.

O projeto foi aprovado em 07 de março de 2018 pelo Comitê de Ética em Pesquisa da instituição, sob protocolo 2.531.761 e CAAE 81997517.3.0000.5259, obedecendo aos preceitos éticos vigentes. Obteve, também, autorização da instituição envolvida para sua realização. Com a assinatura do Termo de Consentimento Livre e Esclarecido, todos os participantes foram informados sobre os objetivos do estudo e a garantia de sigilo individual dos participantes. Foram adotados cuidados para que não passassem por qualquer tipo de constrangimento e não houve compensação financeira ou material pela participação.

\section{Resultados}

Entre os 47 participantes, a maioria era do sexo feminino $(76,6 \%)$, com idade média de 39,47 anos $(\mathrm{DP}=8,24)$. Quanto à categoria profissional, houve predomínio de técnicos de enfermagem $(89,4 \%)$, com tempo médio de exercício de 16,8 anos ( $\mathrm{DP}=7,28)$. Houve predomínio de participantes do plantão diurno (65,9\%). 


\section{Avaliação do conhecimento sobre prevenção e cuidados com lesões por pressão}

Os dados relativos à avaliação utilizando o Instrumento para Identificação do Nível de Conhecimento Sobre Úlcera de Pressão e Medidas Preventivas, são apresentados na Tabela 1, que demonstra os índices de acertos referentes aos resultados relevantes sobre os conhecimentos avaliados, com questões agrupadas de acordo com o conteúdo avaliado, ou apresentadas individualmente, no caso de resultados relevantes a este estudo. Buscou-se também investigar se houve associação entre o conhecimento e práticas avaliados, com as categorias profissionais e unidades de trabalho.

Tabela 1: Teste Qui-quadrado de associação entre as respostas às questões do Instrumento para identificação do nível de conhecimento sobre lesão por pressão: medidas preventivas, categoria profissional e unidade de trabalho $(\mathrm{n}=47)$, Rio de Janeiro-RJ, 2018.

\begin{tabular}{|c|c|c|c|c|}
\hline \multirow[t]{2}{*}{ Conhecimento avaliado (Questão) } & \multicolumn{2}{|c|}{$\begin{array}{c}\text { Resposta } \\
\text { correta }\end{array}$} & \multirow{2}{*}{$\begin{array}{c}\text { p-valor }^{*} \\
\text { Categoria } \\
\text { profissional }\end{array}$} & \multirow{2}{*}{$\begin{array}{l}\text { p-valor } \\
\text { Unidade } \\
\text { Clínica }\end{array}$} \\
\hline & $\mathbf{N}$ & $\%$ & & \\
\hline \multicolumn{5}{|c|}{ Conhecimento avaliado (grupo de questões) } \\
\hline Avaliação da pele & 47 & 100,0 & - & - \\
\hline Avaliação e limpeza da pele & 43 & 91,5 & 0,505 & 0,571 \\
\hline Avaliação dos riscos associados à LP & 45 & 95,8 & 0,673 & 0,477 \\
\hline Medidas preventivas à LP & 43 & 91,9 & 0,422 & 0,336 \\
\hline Mobilização do paciente & 43 & 91,5 & 0,505 & 0,443 \\
\hline Classificação e estadiamento das lesões & 40 & 85,8 & 0,573 & 0,318 \\
\hline \multicolumn{5}{|l|}{ Questões isoladas } \\
\hline Orientação do paciente & 47 & 100,0 & - & - \\
\hline Proteção de proeminências ósseas & 45 & 95,7 & 0,618 & 0,108 \\
\hline Cuidados com a umidade na pele & 43 & 91,5 & 0,670 & 0,253 \\
\hline Fator nutricional do paciente & 42 & 89,3 & 0,717 & 0,632 \\
\hline Redução da pressão com elevação dos calcânceos & 41 & 87,2 & 0,011 & 0,250 \\
\hline $\begin{array}{l}\text { Uso de almofadas de proteção para o paciente na posição } \\
\text { sentada }\end{array}$ & 38 & 80,9 & 0,025 & 0,138 \\
\hline Uso de colchão redutor de pressão & 35 & 74,5 & 0,003 & 0,193 \\
\hline Inspeção sistemática da pele & 29 & 61,7 & 0,291 & 0,834 \\
\hline Classificação dos estágios da lesão & 29 & 61,7 & 0,619 & 0,781 \\
\hline Baixo grau de elevação da cabeceira da cama & 21 & 44,6 & 0,025 & 0,709 \\
\hline Uso de coberturas como medida preventiva & 9 & 19,1 & 0,058 & 0,363 \\
\hline
\end{tabular}

Fonte: dados da pesquisa (2018) 
Conhecimentos e práticas de enfermagem na prevenção e cuidado às lesões por pressão 8

Nas questões referentes à avaliação da pele e à orientação ao paciente e familiar dos riscos a LP, todos os participantes obtiveram 100\% de acertos. Observa-se mais de $80 \%$ de acertos em dezenove itens do instrumento relacionados à avaliação e limpeza da pele, classificação das lesões, proteção de proeminências ósseas, medidas preventivas a LP, mobilização do paciente. Aproximadamente $60 \%$ de respostas corretas referentes inspeção da pele, classificação dos estágios da lesão.

Os índices de acertos reduzem nas questões relacionadas a coberturas protetoras da pele contra fricção $(70,3 \%)$ e medidas preventivas (mais de 50\%). Além disso, mais de 50\% dos participantes apresentaram padrão de resposta incorreta ou declaração de não saber a resposta sobre tempo da mudança de decúbito em pacientes enquanto sentado, classificações das LP, tipos de coberturas protetoras.

Verificou-se que houve a associação entre o padrão de resposta e a categoria profissional dos participantes. Identificou-se também em três questões sobre utilização de equipamentos, mudança de decúbito, predomínio de respostas certas entre técnicos de enfermagem $(\mathrm{p}<0,05)$. Já para duas questões relativas ao posicionamento do paciente no leito e aos tipos de coberturas utilizadas na pele para evitar a fricção, houve predomínio de erros entres os técnicos de enfermagem $(\mathrm{p}<0,05)$.

No cruzamento entre as três unidades e as respostas dos profissionais, não foi observada associação entre o padrão de resposta e a unidade onde trabalhavam.

A partir dos dados qualitativos, observa-se nas falas dos participantes a identificação dos elementos relacionados ao conhecimento e também à rotina de trabalho de enfermagem que favorece o desenvolvimento das lesões por pressão em pacientes internados em unidades de internação clínica. 


\section{Medidas preventivas de lesões por pressão}

Esta categoria apresenta o conhecimento dos profissionais de enfermagem sobre as medidas preventivas a LP. A categoria abarcou um total de oito unidades de significação e 72 UR, as unidades de significação que se destacaram foram "pele seca” e "mobilização do paciente no leito" ambas com 19 UR (11,4\%), seguido de "higiene corporal do paciente” com 10 UR (6,0\%).

Pacientes em uso de fraldas a gente pede sempre para manter esse paciente seco. (Enf.01)

Esses pacientes que estão dependentes da enfermagem precisam de mais cuidados em trocas constantes de fraldas. (Tec.09)

A gente tenta é aumentar o número de mudanças de decúbito, que também é o utópico que a gente sabe que não vai conseguir, mas a gente tenta (Enf.05)

Então, devemos estar mudando o paciente sempre de posição, mudança de decúbito de 2 em 2 horas ou próximo disso (Enf.07)

Demais medidas preventivas citadas foram os temas "higiene corporal do paciente" com dez UR’s (6\%) e “pele bem hidratada”, com quatro UR’s $(2,4 \%)$.

É manter a pele sempre higienizada. (Tec. 09)

Hidratação da pele isso dá para fazer com o que a gente tem aqui, creme de uréia, com óxido de zinco enfim a gente faz isso também. (Enf. 05)

A mudança de decúbito foi a medida preventiva mais citada nas entrevistas. Neste tema “mobilização do paciente no leito” surgiram 19 UR (11,4\%), conforme os depoimentos.

A gente tenta é aumentar o número de mudanças de decúbito, a gente sabe que não vai conseguir, mas a gente tenta. (Enf. 05)

A gente não consegue fazer a rotina de mudança de decúbito de 2 em 2 horas. (Tec. 11) 


\section{Fatores que influenciam no aparecimento de lesões por pressão}

Surgiram nessa categoria quatro unidades de significação, em um total de 37 unidades de registro. As unidades que se destacaram foram "condições individuais do paciente" com 20 UR (12,6\%), e “déficit de profissionais na unidade de clínica médica” com 10 UR’S (6,0\%).

Como medidas preventivas na área de enfermagem, a gente enfoca as questôes relacionadas à mudança de posição de maneira individualizada. É porque cada paciente tem sua necessidade. (Enf. 01)

Aqui na enfermaria não tem rotina como em setor fechado por não ter o número de profissionais para fazer. Aqui na enfermaria é sempre cheio e paciente acamado, com poucos profissionais. (Enf.11)

Sempre que possivel a gente tenta fazer, seguir essas normas, porém, nem sempre é possível, devido ao nosso quadro deficitário de profissionais que acaba implicando de forma negativa na assistência de enfermagem. (Enf.07)

O raciocínio clínico do enfermeiro foi identificado indiretamente nas falas, sendo essencial para determinar as etapas deste processo, que inclui a avaliação dos pacientes.

Paciente que tem risco a lesão por pressão, são pacientes comatosos, ou torporosos, nível de consciência rebaixado, desnutrido, caquético, paciente com dificuldade em movimentação, é isso são os pacientes que normalmente correm mais risco de abrir lesão por pressão, paciente que tenham alguma doença dermatológica, e principalmente em uso de aminas que tem também influência a esse tipo agravo. (Enf.09)

\section{Discussão}

Como proposto, foi possível investigar os conhecimentos e práticas de enfermeiros e técnicos de enfermagem de três unidades de internação clínica sobre a prevenção e cuidados com as LP.

Da mesma maneira que o observado neste estudo, a enfermagem é uma profissão que historicamente desde seu surgimento foi constituída pelo sexo feminino, seja pela a tradição cultural ou por demais fatores que contribuiram para a feminilização nesta área da saúde. Dados 
da pesquisa sobre o perfil da enfermagem brasileira confirmam que a equipe de enfermagem ainda é predominantemente feminina $(85,1 \%)$, em pleno rejuvenescimento pelo fato da existência de inúmeras faixas etárias e pelo crescimento de recém-formados, com predomínio de técnicos de enfermagem devido às características da divisão do trabalho. ${ }^{15}$

Mundialmente, verifica-se que há preocupação sobre o conhecimento dos profissionais acerca de medidas preventivas de LP, reconhecido como abaixo do esperado em diferentes estudos. ${ }^{16-17}$

Entre as medidas preventivas destaca-se que, além da avaliação dos fatores de risco, cuidados como inspeção diária da pele, reposicionamento do paciente e a higiene da pele são relevantes. Uma série de estratégias podem ser adotadas, mas as ações desenvolvidas dependem do conhecimento dos profissionais de enfermagem para efetivar uma boa conduta no cuidado. ${ }^{18-19}$

Os profissionais de enfermagem reconhecem a mudança de decúbito como a principal medida de prevenção à LP, além da importância da manutenção da pele do paciente. Mas citam que esses cuidados são prejudicados devido o quadro deficitário da equipe de enfermagem nas unidades de internação.

Pequenas mudanças na posição incluem mover o paciente no leito e alterar a posição do membro inferior. Estas estratégias ajudam a aliviar a pressão e melhorar a perfusão dos tecidos comprimidos. ${ }^{20}$ Durante a mobilização do paciente no leito é necessário a presença de mais de um profissional, para evitar o efeito da fricção e cisalhamento. A equipe de enfermagem tem dificuldade para realizar a mobilização do paciente devido o número menor de profissionais.

Destaca-se que o incremento de enfermeiros e técnicos de enfermagem tem potencial para reduzir significativamente índices de afastamentos por doença, banco de horas excedentes, pagamento de horas extras, bem como reduzir as taxas de lesão por pressão, quedas e infecções relacionadas ao uso de sonda vesical de demora. ${ }^{21}$ Ainda assim, não há publicações ou 
Conhecimentos e práticas de enfermagem na prevenção e cuidado às lesões por pressão| 12

recomendações disponíveis sobre o quantitativo adequado de recursos humanos considerando a prevenção da LP. ${ }^{22}$

Ratifica-se a necessidade de atualização profissional, ainda que no contexto de dimensionamento desfavorável. O uso correto do conceito e terminologia apresentados pelo NPUAP em 2016, faz com que os profissionais de saúde possam avaliar e desenvolver estratégias de enfrentamento de maneira mais efetiva., ${ }^{1,23}$

A literatura descreve que quanto aos cuidados diretos com a pele, a mudança de decúbito é relatada usualmente em ações de prevenção, perdendo atenção quando a lesão já está instalada. Neste momento, ganha prioridade a realização de curativos. A higienização do paciente realizada diariamente pela equipe de enfermagem, é uma das medidas reconhecida por proporcionar conforto. $^{24,25}$ Assim, verificam-se que ações de prevenção, que devem ser continuadas, a fim de evitar novas lesões ou complicações existentes, são abandonadas em determinado momento da assistência.

Sabe-se que existem fatores extrínsecos e intrínsecos que estão associados ao estado de saúde do paciente, o que indica a instauração de cuidados individualizados. A especificidade dos cuidados implementados deve ser avaliada individualmente, conforme os fatores de risco a LP que o paciente possui. ${ }^{20,25}$

As lesões são fenômenos complexos e a prevenção e cuidados relacionados exige conhecimento baseado em evidências e um conjunto de medidas por parte do profissional, porém também demanda políticas de gestão e educação como iniciativas da própria instituição, com recursos humanos e materiais que viabilizem uma assistência segura. ${ }^{25}$

Por fim, infere-se que as questões que apresentaram maior número de acertos estão relacionadas a cuidados realizados diariamente e que exigem menor conhecimento científico e 
tecnológico. Os dados obtidos demonstram deficiência no conhecimento da equipe de enfermagem sobre as novas tecnologias utilizadas como medidas preventivas a LP.

Ressalta-se a importância do incentivo a capacitação dos profissionais, no intuito de fortalecimento do conhecimento baseado nas evidências existentes, com o objetivo de melhoria na qualidade da assistência.

\section{Conclusão}

Apesar da temática ser amplamente estudada e discutida no meio científico, os resultados permitiram identificar que ainda existe deficiência no conhecimento da equipe de enfermagem sobre os múltiplos fatores que predispõem a LP, incluindo as novas nomenclaturas, classificação das lesões e incorporação de novas tecnologias do cuidado.

Em relação ao instrumento utilizado, as respostas corretas estavam de acordo com as medidas básicas de prevenção de LP como limpeza e inspeção da pele. No entanto, desconhecem coberturas para a proteção da pele contra fricção. A entrevista corroborou com os resultados encontrados na primeira etapa e eles citam que as condições individuais do paciente e o déficit de profissionais de enfermagem contribuem para a ocorrência de LP.

Assim, de acordo com os achados, destaca-se a necessidade de implementação de medidas de educação permanente em serviço para reforçar conteúdos sobre o tema, além de aproximar os profissionais das mudanças e novos conhecimentos baseados em evidências científicas que passem a ser incorporados por este campo de conhecimento.

Para contribuir com o campo de estudo, foi encaminhado um relatório com os resultados obtidos ao Núcleo de Segurança do Paciente da instituição, para auxiliar no planejamento das ações educativas. 
Conhecimentos e práticas de enfermagem na prevenção e cuidado às lesões por pressão | 14

No que se refere a limitação do estudo, o número reduzido de profissionais de enfermagem nas clínicas médicas do hospital, principalmente relacionados ao plantão noturno, somado a ausência de participantes devido a férias, licença ou recusa na participação, foi considerada prejudicial. É importante destacar que a instabilidade política no Estado do Rio de Janeiro e os sucessivos cortes no orçamento do hospital, contribuem negativamente na aquisição de recursos humanos, não só da equipe de enfermagem, mas também no corpo de trabalhadores de forma geral.

\section{Referências}

1. National Pressure Ulcer Advisory Panel (NPUAP). NPUAP pressure injury stages [Internet]. Washington (DC); 2016 [acesso em 2017 maio 10]. Disponível em: https://npuap.org/page/PressureInjuryStages

2. Associação Brasileira de Estomaterapia (SOBEST). Classificação das lesões por pressão: consenso NPUAP 2016 - Adaptada culturalmente para o Brasil. São Paulo; 2016 [acesso em 2019 ago 28]. Disponível em: http://www.sobest.org.br/textod/35

3. Quirino DES, Faustino AM, Freitas RO, Oliveira AB, Medved IV. Fatores de risco para desenvolvimento de úlcera por pressão em unidade de internação clínica. Estima [Internet]. 2014 [acesso em 2017 jul 10];15(1):1-17. Disponível em: https://www.revistaestima.com.br/index.php/estima/article/view/99

4. Laranjeira CA, Loureiro S. Fatores de risco das úlceras de pressão em doentes internados num hospital português. Rev Salud Pública [Internet]. 2017 [acesso em 2017 jul 10];19(1):161-70. Disponível em: https://doi.org/10.15446/rsap.v19n1.42251

5. Neiva GP, Carnevalli JR, Cataldi RL, Furtado DM, Fabri RL, Silva PS. Alterações dos parâmetros hematológicos em pacientes portadores de úlcera por pressão em um hospital de longa permanência. Einsten [Internet]. 2014 [acesso em 2017 jul 10];12(3):304-9. Disponível em: http://www.scielo.br/pdf/eins/v12n3/pt_1679-4508-eins-12-3-0304.pdf

6. Galvão NS, Serique MAB, Santos VLCG, Nogueira PC. Conhecimentos da equipe de enfermagem sobre prevenção de úlceras por pressão. Rev Bras Enferm [Internet]. 2017 [acesso em 2017 jul 10];70(2):312-8. Disponível em: http://www.scielo.br/pdf/reben/v70n2/pt_0034-7167-reben-70-02-0294.pdf

7. Cedraz RO, Gallasch CH, Pérez Júnior EF, Gomes HF, Rocha RG, Mininel VA. Risks management in the hospital environment: incidence and risk factors associated with falls and pressure injuries in a 
clinical unit. Esc Anna Nery Rev Enferm [Internet]. 2018 [acesso em 2017 jul 10];22(1):e20170252. Disponível em: http://dx.doi.org/10.1590/2177-9465-ean-2017-0252

8. Jesus JAP, Machado DG, Almeida JS, Rodrigues APB, Maranhão TA, Silva GB. Saberes e práticas de enfermeiros sobre a prevenção de lesão por pressão. Rev Prev Infecç Saúde [Internet]. 2018 [acesso em 2019 ago 28];4:7771. Disponível em: http://www.ojs.ufpi.br/index.php/nupcis/article/download/7771/pdf_1

9. Mattos RM, Melo FBS, Araújo AKC, Gomes GMS, Vasconcelos LDS, Souza LDT. Educação em saúde aos trabalhadores de enfermagem e acompanhantes sobre prevenção e tratamento de lesões de pele em dois hospitais de Petrolina-PE. Interfaces [Internet]. 2015 [acesso 2019 ago 28];3(1):22-32. Disponível em https://www.ufmg.br/proex/revistainterfaces/index.php/IREXT/article/view/44/pdf

10. Ministério da Saúde (BR), Agência Nacional de Vigilância Sanitária. Assistência segura: Uma reflexão teórica aplicada à prática [Internet]. Brasília (DF): Ministério da Saúde; 2013 [acesso em 2019 ago 28]. Disponível em: http://portal.anvisa.gov.br/documents/33852/3507912/Caderno+1++Assistencia+Segura+-+Uma+Reflexao+Teorica+Aplicada+a+Pratica/97881798-cea0-4974-9d9b077528 ea1573

11. Santos JLG, Erdamnn AL, Meirelles BHS, Lanzoni GMM, Cunha VP, Ross R. Integração entre dados quantitativos e qualitativos em uma pesquisa de métodos mistos. Texto \& Contexto Enferm [Internet]. 2017 [acesso em 2019 out 22]. Disponível em: http://dx.doi.org/10.1590/010407072017001590016

12. Fernandes LM. Efeitos de intervenções educativas no conhecimento e práticas de profissionais de enfermagem na incidência de úlceras de pressão em Centro de Terapia Intensiva [tese]. São Paulo (SP): Universidade de São Paulo; 2006. 238 p.

13. Pieper B, Mott M. Nurse's knowledge of pressure ulcer prevention, staging and description. Adv Wound Care. 1995;8(3):38-40.

14. Bardin L. Análise de conteúdo. São Paulo: Edições 70; 2011. 229 p.

15. Machado MH. Aguiar Filho W, Lacerda WF, Oliveira E, Lemos W, Wermelinger M, et al. Características gerais da enfermagem: o perfil sociodemográfico. Enferm Foco (Brasília) [Internet]. 2016 [acesso em 2018 out 01];7(S1):9-14. Disponível: http://revista.cofen.gov.br/index.php/enfermagem/article/view/686/0

16. Fulbrook P, Lawrence $P$, Miles S. Australian nurses' knowledge of pressure injury prevention and management: a cross-sectional survey. J Wound Ostomy Continence Nurs [Internet]. 2019 [acesso em 2019 ago 28];46(2):106-12. Disponível em: https://www.ncbi.nlm.nih.gov/pubmed/30801563

17. Dalvand S, Ebadi A, Gheshlagh RG. Nurses' knowledge on pressure injury prevention: a systematic review and meta-analysis based on the Pressure Ulcer Knowledge Assessment Tool. Clin Cosmet 
Conhecimentos e práticas de enfermagem na prevenção e cuidado às lesões por pressão| 16

Investig Dermatol [Internet]. 2018 [acesso em 2019 ago 28];11:612-20. Disponível em: https://www.ncbi.nlm.nih.gov/pmc/articles/PMC6257136/pdf/ccid-11-613.pdf

18. Brandão ES, Mandelbaum MHS, Santos I. Um desafio no cuidado em enfermagem: prevenir úlceras por pressão no cliente. Rev Pesqui Cuid Fundam [Internet]. 2013 [acesso em 2019 ago 28];5(1):3221-8. Disponível em: https:/www.redalyc.org/articulo.oa?id=505750897041

19. Gomes FSL, Bastos MAR, Matozinhos FP, Temponi HR, Velásquez-Meléndez G. Avaliação de risco para úlcera por pressão em pacientes críticos. Rev Esc Enferm USP [Internet]. 2011 [acesso em 2018 ago 20];45(2):313-8. Disponível em: http://www.scielo.br/scielo.php?script=sci_arttext\&pid=S0080$62342011000200002 \& \operatorname{lng}=\mathrm{pt} \& \operatorname{lng}=\mathrm{pt}$

20. Silva MLN, Caminha RTÓ, Oliveira SHS, Diniz ERS, Oliveira JL, Neves VSN. Úlcera por pressão em unidade de terapia intensiva: análise da incidência e lesões instaladas. Rev RENE [Internet]. 2013 [acesso em 2018 julho 10];14(5):938-44. Disponível em: http://www.periodicos.ufc.br/rene/article/view/3623

21. Quadros DV, Magalhães AMM, Mantovani VM, Rosa DS, Echer IC. Análise de indicadores gerenciais e assistenciais após adequação de pessoal de enfermagem. Rev Bras Enferm [Internet]. 2016 [acesso em 2017 jan 18];69(4):684-90. Disponível em: http://dx.doi.org/10.1590/0034-7167.2016690410i

22. Tonole R, Brandão ES. Recursos humanos e materiais para prevenção de lesão por pressão. Rev Enferm UFPE On Line [Internet]. 2018 [acesso em 2019 ago 28];12(8):2170-80. Disponível em: https://periodicos.ufpe.br/revistas/revistaenfermagem/article/view/235091

23. Moraes JT, Borges EL, Lisboa CR, Cordeiro DCO, Rosa EG, Rocha NA. Conceito e classificação de lesão por pressão: atualização do National Pressure Ulcer Advisory Panel. Rev Enferm Cent Oeste Min [Internet]. 2016 [acesso em 2019 ago 28];6(2):2292-306. Disponível em: http://www.seer.ufsj.edu.br/index.php/recom/article/view/1423

24. Menezes LCG, Fernandes MMJ, Guedes MVC, Oliveira RM, Leitão IMTA, Moura DJM. Cuidados clínicos e gerenciais de enfermagem na prevenção de úlcera por pressão. Estima [Internet]. 2017 [acesso em 2019 ago 28];15(2):107-14. Disponível em: https://www.revistaestima.com.br/index.php/estima/article/view/486

25. Tonole R, Brandão ES. Recursos humanos e materiais para a prevenção da lesão por pressão. Rev enferm UERJ[Internet]. 2019 [acesso em 2019 nov 12];27:e38742. Disponível em: https://www.epublicacoes.uerj.br/index.php/enfermagemuerj/article/view/38472/29906 


\section{Autor correspondente}

Cristiane Helena Gallasch

E-mail: cristiane.gallasch@gmail.com

Endereço: Blv 28 de Setembro, 157, $7^{\circ}$ andar

CEP: 20551-030

\section{Contribuições de Autoria}

\section{1 - Francisca Débora Veras Silva}

Concepção e planejamento do projeto de pesquisa; obtenção, análise e interpretação de dados, redação e revisão crítica do manuscrito.

\section{2 - Priscila Cristina da Silva Thiengo de Andrade}

Concepção e planejamento do projeto de pesquisa; obtenção, análise e interpretação de dados, redação e revisão crítica do manuscrito.

\section{3 - Eugenio Fuentes Perez Júnior}

Análise e interpretação de dados, redação e revisão crítica do manuscrito.

\section{4 - Ariane da Silva Pires}

Análise e interpretação de dados, redação e revisão crítica do manuscrito.

\section{5 - Cristiane Helena Gallasch}

Concepção e planejamento do projeto de pesquisa; obtenção, análise e interpretação de dados, redação e revisão crítica do manuscrito.

\section{Como citar este artigo}

Silva FDV; Andrade PCST, Pérez Júnior EFP, Pires AS, Gallasch CH. Conhecimentos e práticas de enfermagem na prevenção e cuidados às lesões por pressão. Rev. Enferm. UFSM. 2019 [Acesso em: Anos Mês Dia]; vol.9 e:1-18. DOI:https://doi.org/10.5902/2179769238804 